J. Lake Sci. (湖泊科学) , 2016, 28(6): 1185-1193

DOI 10. 18307/2016. 0604

(C) 2016 by Journal of Lake Sciences

\title{
湖泊疏浚堆场淤泥污染及潜在生态风险评价
}

\author{
刘 敏 ${ }^{1,2}$, 钟继承 ${ }^{1 * *}$, 余居华 ${ }^{1,2}$, 郑小兰 ${ }^{1,2}$, 范成新 ${ }^{1}$ \\ (1: 中国科学院南京地理与湖泊研究所湖泊与环境国家重点实验室, 南京 210008) \\ (2: 中国科学院大学, 北京 100049)
}

\begin{abstract}
摘 要: 疏浚淤泥内通常含有不同类型的有毒有害物质,在堆场直接堆放过程中可能会对周围环境产生有害影响. 本文 针对太湖及巢湖相应疏浚堆场内淤泥进行研究, 探讨淤泥中重金属、多环芳烃以及多氯联苯等污染物含量及潜在生态风 险; 根据重金属的风险指数法和持久性有机污染物的风险商法,对各污染物的潜在生态风险进行定量分析. 研究结果表 明, 太湖白施堆场以及孔湾堆场淤泥内重金属及多环芳烃含量较小, 潜在生态风险较低; 巢湖南庄堆场淤泥内各类有害 物质含量较大, 种类较多, 对于周围环境具有较高的潜在生态威胁. 多氯联苯则在各个疏浚堆场淤泥中具有很高的积累 量,潜在生态风险较高,应引起管理者的重视.
\end{abstract}

关键词 : 疏浚堆场; 疏浚底泥; 重金属;多环芳烃;多氯联苯;潜在生态风险

\section{Distribution and potential ecological risk assessments of contaminants insludge in dredged sediments landfill sites}

\author{
LIU Min $^{1,2}$, ZHONG Jicheng ${ }^{1 * *}$, YU Juhua ${ }^{1,2}$, ZHENG Xiaolan ${ }^{1,2}$ \& FAN Chengxin ${ }^{1}$ \\ (1: State Key Laboratory of Lake Science and Environment, Nanjing Institute of Geography and Limnology, Chinese Academy \\ of Sciences, Nanjing 210008, P.R.China) \\ (2: University of Chinese Academy of Sciences, Beijing 100049, P.R.China)
}

\begin{abstract}
Direct disposal of dredged sediments in landfill sites may possess potential ecological risks to environment as they are abundant in contaminant. An experiment was conducted to analysis the concentrations and potential ecological risks of different contaminants including heavy metals, polycyclic aromatic hydrocarbons (PAHs) and polychlorinated biphenyls (PCBs) in dredged sediment landfill sites in Lake Taihu and Lake Chaohu in China. According to the potential ecological risk assessments of heavy metals and risk quotients of persistent organic pollutants, the potential ecological risks of different contaminants were quantified. Results illustrate that potential ecological risks of dredged sediments are relatively low in landfill sites in Lake Taihu, while are considerable in Lake Chaohu. In addition, the high accumulations of PCBs in dredged sediments possess high potential ecological risks to environment, and need more attention from managers.
\end{abstract}

Keywords: Dredged sediment; landfill site; heavy metal; polycyclic aromatic hydrocarbon; polychlorinated biphenyl; potential ecological risk

近年来, 底泥疏浚被广泛地应用于航道疏通 ${ }^{[1-2]}$ 、湖泊修复 ${ }^{[3-6]}$ 和渔业应用等各方面. 底泥疏浚能有效地 去除富含污染物质的湖泊底泥, 减少湖泊内源营养负荷, 降低有毒有害物质对水体的潜在危害. 然而,如何 对疏浚过程中产生的淤泥进行处置仍然是一大难题. 疏浚产生的淤泥目前主要是堆放在疏浚堆场中, 单纯 地将其进行堆放而不采取其他的控制措施会带来各种环境问题. 首先, 底泥疏浚产生的淤泥量巨大, 需要大 量的土地来堆放. 如, 上海仅对苏州河的治理就需疏浚淤泥数十万立方米, 珠江三角洲地带每年疏浚的淤泥

* 国家水体污染控制与治理科技重大专项 (2013ZX07113001, 2012ZX07101-010) 和国家自然科学基金项目 (41171367,41371457) 联合资助. 2016-01-24 收稿; 2016-05-25 收修改稿. 刘敏 (1992 ), 男, 硕士研究生; E-mail: liumin10480_cpu@163.com.

** 通信作者;E-mail: jczhong@ niglas.ac.cn. 
可达到 $8000 \times 10^{4} \mathrm{~m}^{3[7]}$; 其次, 淤泥内含有重金属、持久性有机污染物等, 若不对其进行及时处理,这些污染 物将有可能会渗透进人地下水或随表面径流进人周围水体, 产生二次污染; 相反, 由于底泥中含有丰富的 氮、磷等营养元素 ${ }^{[8]}$, 若经合理应用的话可改善土壤营养结构, 增加农作物产量 ${ }^{[9]}$. 因此, 探索疏浚淤泥的资 源化利用途径便显得尤为重要. 而在将疏浚淤泥进行资源化利用之前, 有必要对疏浚堆场内的淤泥性质进 行研究,探究淤泥的污染状况,定量评价其潜在生态风险,为后续的资源化利用提供依据.

疏浚淤泥中通常含有不同类型的有毒有害物质,包括重金属、多环芳烃和多氯联苯等,在堆场堆放的过 程中可能会对周围环境产生影响. 其中重金属是一类不能被微生物降解, 而易于通过食物链的放大作用在 环境中累积的污染物, 高含量的重金属可以使生物体内的蛋白质和酶失活, 从而对人类及水体生态系统产 生极大的危害作用. 多环芳烃是煤、石油等物质经不完全燃烧所产生的半挥发性有机物, 是强烈的致癌物 质, 可以通过呼吸或者皮肤接触等方式进人人体而对人体产生危害. 美国 $\mathrm{EPA}^{[10]}$ 规定了 16 种优先控制多环

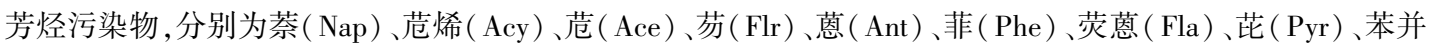
$[\mathrm{a}]$ 葱 $(\mathrm{BaA}) 、$ 营 $(\mathrm{Chr}) 、$ 苯并 $[\mathrm{b}]$ 荧葱 $(\mathrm{BbF}) 、$ 苯并 $[\mathrm{k}]$ 荧葱 $(\mathrm{BkF}) 、$ 苯并 $[\mathrm{a}]$ 芘 $(\mathrm{BaP}) 、$ 狮并 $[1,2,3 \mathrm{~cd}]$ 芘 $(\mathrm{InP})$ 、二苯并 $[\mathrm{a}, \mathrm{h}]$ 葱 $(\mathrm{DahA})$ 和苯并 $[\mathrm{g}, \mathrm{h}, \mathrm{i}]$ 芘 $(\mathrm{BghiP})$. 多氯联苯极难被降解, 能够在生物体内大量富集, 具有强烈的致癌、致畸、致突变等效应.

近年来, 关于堆场淤泥内污染物的生态风险研究已有报道, 如 Stephens 对河道淤泥堆场内重金属的淋 溶特性进行研究 ${ }^{[11]}$, 发现在淤泥脱水及氧化过程中, 重金属 $\mathrm{Ni} 、 \mathrm{Zn} 、 \mathrm{~Pb} 、 \mathrm{Cr} 、 \mathrm{Cu}$ 将由残渣态向可还原态或可 氧化态进行转化, 从而使得各金属的淋溶特性增强,导致堆场淤泥内重金属对周围地下水、地表水构成潜在 威胁; R $\mu$ mney ${ }^{[12]}$ 则研究了堆场及堆场周围淤泥内多环芳烃含量及其生态风险, 发现疏浚堆场淤泥内多环芳 烃含量较高, 同时多环芳烃有向周围土壤扩散的趋势, 从而对堆场周围生态环境产生潜在危害; 张路等 ${ }^{\left[{ }^{[13}\right]}$ 的 研究表明, 在堆场使用前后, 土壤内多环芳烃含量虽然未发生明显变化, 但堆场内生长的植物一水花生体内 多环芳烃含量明显增加; 李涛等 ${ }^{[14]}$ 发现堆场下部黏土层对疏浚底泥中的污染物总氮、总磷及 COD 等具有较 强的吸附和阻隔能力. 本文针对太湖及巢湖相关湖区疏浚淤泥堆场开展研究, 目的是通过对疏浚堆场淤泥 中有毒有害物质进行分析, 探究堆场淤泥中所含污染物的污染状况, 评价各类污染物的潜在生态风险, 为疏 浚堆场底泥后续的资源化利用提供相关依据,同时也能为疏浚堆场淤泥堆放环境管理提供参考依据.

\section{1 材料与方法}

\section{1 研究区域概况}

太湖 $\left(30^{\circ} 55^{\prime} 40^{\prime \prime} \sim 31^{\circ} 32^{\prime} 58^{\prime \prime} \mathrm{N}, 119^{\circ} 52^{\prime} 32^{\prime \prime} \sim 120^{\circ} 36^{\prime} 10^{\prime \prime} \mathrm{E}\right)$ 和巢湖 $\left(31^{\circ} 22^{\prime} \sim 32^{\circ} 03^{\prime} \mathrm{N}, 118^{\circ} 04^{\prime} \sim 118^{\circ} 29^{\prime} \mathrm{E}\right)$ 分别位于江苏及安徽省境内, 是我国第三和第五大淡水湖泊, 是其周围流域重要的水源地. 近几十年来, 由 于经济的迅速发展及人口的快速增长 2 个湖泊均出现了严重的富营养化, 尤其是太湖北部的梅梁湾地区频 繁暴发蓝藻水华. 湖泊富营养化严重制约了湖泊的生态服务功能 ${ }^{[5-16]}$, 因此, 对富营养化湖泊进行生态修复 特别是淤泥清除具有重大的现实意义和经济价值. 本研究选取江苏太湖 2009 年以来梅梁湾地区疏浚底泥 堆放场地白施堆场、孔湾堆场淤泥及安徽巢湖地区“十二五”水专项巢湖项目疏浚示范区南庄疏浚堆场淤泥 作为研究对象. 相关堆场的疏浚工作已经结束, 目前底泥主要堆放在堆场等待进一步的处理. 太湖梅梁湾紧 靠无锡、常州等工业相对发达地区, 周围乡镇企业较多 ${ }^{[17]}$; 巢湖水域同样也是接受了大量来自合肥等市区的 工农业废水及生活污水 ${ }^{[18]}$, 各类工业如纺织、机械电子等行业废水及生活污水的排放使得大量污染物质进 人水体, 经一系列迁移转化最终进人水底沉积物. 沉积物经疏浚后, 由于周围氧化还原条件的变化, 这些污 染物质就有可能再释放重新进人水体、土壤及相关生态系统.

\section{2 样品采集与分析}

在太湖白施堆场、太湖孔湾堆场以及巢湖南庄堆场各设置 1 个疏浚淤泥采样点, 样品采集时, 为确保样 品代表性, 在堆场内用采样工具进行多点采样, 把采集的淤泥样品进行充分混合后, 取适量的淤泥带回实验 室进行后续的处理和分析.

样品冷冻干燥后进行研磨过䇥, 在研磨和过篮时应尽量避免处理过程中可能对样品的污染问题, 用于 重金属分析的样品用玛瑙研钵研磨, 用塑料篮进行篮分; 而用于持久性有机物分析的样品采用玛瑙研钵研 
磨, 用金属篮网进行篮分. 淤泥 $\mathrm{pH}$ 、容重、含水率、有机碳及烧失量的测定依照《土壤农业化学分析方法》 ${ }^{[19]}$ 进行; 淤泥粒径则采用激光粒度分析仪 (Mastersize 2000) 进行测定.

由于淤泥内各类有机及无机污染物种类较多, 成分复杂, 不可能考虑到每一种污染物质的分布情况, 因 此仅选择具有代表性的 8 种毒性较大的重金属镉 $(\mathrm{Cd})$ 、镍 $(\mathrm{Ni})$ 、铜 $(\mathrm{Cu})$ 、锌 $(\mathrm{Zn})$ 、砷 $(\mathrm{As}) 、$ 、铅 $(\mathrm{Pb})$ 、铬 $(\mathrm{Cr})$ 和录 $(\mathrm{Hg})$ 污染物、16 种优先控制多环芳烃以及多氯联苯等持久性有机污染物进行分析. 其中淤泥样品经 $\mathrm{HNO}_{3}-\mathrm{HF}-\mathrm{HClO}_{4}$ 消化法消解后, 采用电感耦合等离子体质谱仪 ( ICP-MS) 测定 $\mathrm{Cd} 、 \mathrm{Ni} 、 \mathrm{Cu} 、 \mathrm{Zn} 、 \mathrm{As} 、 \mathrm{~Pb} 、 \mathrm{Cr}$ 含 量, $\mathrm{Hg}$ 含量的测定则采用全自动固体测永仪 ${ }^{[20]}$.

16 种优先控制多环芳烃含量的测定采用气相色谱一质谱联用技术 (GC-MS) : 准确称取干燥的 $5 \sim 20 \mathrm{~g}$ 淤 泥样品于三角烧瓶中, 加人 $50 \mathrm{ml}$ 二氯甲烷, 超声萃取 $1 \mathrm{~h}$, 离心后移取上层萃取清液, 经重复一次萃取后, 再 用 $10 \mathrm{ml}$ 二氯甲烷洗涤, 合并萃取液于梨形瓶内, 旋转蒸发至 $2.0 \mathrm{ml}$. 采用硅胶柱层析法进行样品纯化. 以二 氯甲烷洗脱 PAHs, 洗脱液收集人 $25 \mathrm{ml}$ 梨形瓶中, 用无水硫酸钠干燥后用旋转蒸发仪浓缩至 $1 \mathrm{ml}$ 左右, 再 用 $\mathrm{N}_{2}$ 缓缓吹扫溶剂至 $0.1 \mathrm{ml}$. 色谱条件: DB-5MS 毛细管柱 $(30 \mathrm{~m} \times 0.25 \mathrm{~mm} \times 0.25 \mu \mathrm{m}$, 美国 J\&W 公司). 流 速: $1 \mathrm{ml} / \mathrm{min}$; 升温程序: $40^{\circ} \mathrm{C}$ 保持 $5 \mathrm{~min}$, 以 $8^{\circ} \mathrm{C} / \mathrm{min}$ 的速率上升到 $280^{\circ} \mathrm{C}$, 保持 $11 \mathrm{~min}$, 不分流进样 $1 \mu \mathrm{l}$. 质 谱条件: 离子源能量 $70 \mathrm{eV}$; 进样口温度: $250^{\circ} \mathrm{C}$; 质量扫描范围: $45 \sim 450 \mathrm{amu}$. 样品分析前, 采用选择性离子法 对 EPA625 混标标样进行检测, 各测定 6 次. 各化合物的精密度在 $2 \% \sim 15 \%$. 配制不同浓度的标样进行分 析, 所得的标准曲线线性关系良好, 相关系数 $r$ 均达到 0.99 , 以仪器 3 倍噪声所对应的浓度作为检测限, 低于 检测限的样品看作未检出 ${ }^{[13]}$.

多氯联苯含量采用气相色谱分析, 淤泥样品用体积比为 $1: 1$ 的正己烷一丙酮提取液浸提、超声提取、离心 后转人复合硅胶柱, 经正已烷洗脱后采用带有电子俘获检测器和自动进样器的 Varian3800 型气相色谱仪分 析. 色谱柱: $\mathrm{CP}-\mathrm{sil} 24 \mathrm{CB}(30 \mathrm{~m} \times 0.25 \mathrm{~mm} \times 0.25 \mu \mathrm{m})$, 进样温度 $260^{\circ} \mathrm{C}$, 检测器温度 $300^{\circ} \mathrm{C}$. 程序升温: 初始温度 $180^{\circ} \mathrm{C}$, 保留 $0.5 \mathrm{~min}, 30^{\circ} \mathrm{C} / \mathrm{min}$ 梯度升温至 $260^{\circ} \mathrm{C}$, 持续 $18 \mathrm{~min}$, 然后 $15^{\circ} \mathrm{C} / \mathrm{min}$ 梯度升温至 $270^{\circ} \mathrm{C}$, 持续 $2 \mathrm{~min}$. 无分流进样 $1 \mu \mathrm{l}$, 载气为高纯氮, 流速为 $1.0 \mathrm{ml} / \mathrm{min}$. 质量控制: 在样品分析过程中进行方法空白、基质加标、 平行样以及加标回收率测定. 21 种 PCBs 混标 ( $10 \mu \mathrm{g} / \mathrm{kg}$ ) 的基质加标平均回收率是 72.0\% 109.8\%, 相对标 准偏差是 $3.13 \% \sim 57.3 \%$, 仪器检测限为 $1.43 \sim 5.10 \mu \mathrm{g} / \mathrm{kg}$, 方法检出限为 $1.33 \sim 3.45 \mu \mathrm{g} / \mathrm{kg}$. 采用七点校正法 得到标准物质的校正曲线, 根据保留时间对目标化合物进行定性分析, 采用峰面积积分法进行定量计算 ${ }^{[21]}$.

每个样品有 3 次平行, 取其平均值进行分析计算. 同时, 各污染物的分布差异采用单因子方差分析进行 评价.

\section{3 重金属的潜在生态风险评价方法}

重金属的潜在生态风险采用 Hakanson 提出的 “潜在生态风险指数法” ${ }^{[22]}$ 进行评价, 重金属的潜在风险 指数定义为:

$$
\begin{gathered}
R I=\sum_{i=1}^{n} E_{r}^{i}=\sum_{i=1}^{n} T_{r}^{i} \cdot C_{f}^{i} \\
C_{d}=\sum C_{f}^{i}=\sum \frac{C_{i}}{C_{f}}
\end{gathered}
$$

式中, $R I$ 为重金属的潜在生态风险指数, $E_{r}^{i}$ 为单一重金属的风险因子, $T_{r}^{i}$ 为重金属的毒性响应因子, 分别设 定为 $\mathrm{Hg}=40, \mathrm{Cr}=2, \mathrm{Ni}=\mathrm{Cu}=\mathrm{Pb}=5, \mathrm{Zn}=1, \mathrm{As}=10, \mathrm{Cd}=30^{[22-23]}, C_{f}^{i}$ 为单一重金属的污染因子, $C_{d}$ 为重金属污 染度, $C_{i}$ 和 $C_{f}$ 分别为重金属浓度实测值及计算所需参比值, 参比值一般选取研究区域所在地区土壤背景 值, 由于太湖白施堆场、孔湾堆场及巢湖南庄堆场分别位于江苏省及安徽省, 因此分别采用江苏省及安徽省 的土壤元素背景值 ${ }^{[24]}$ 作为计算所需的参比值. 重金属污染的潜在生态风险各指标分级标准见表 1.

\section{4 多环芳烃与多氯联苯的潜在生态风险评价方法}

对多环芳烃及多氯联苯可采用风险商 ( risk quotient, $R Q$ ) 进行评价, 根据沉积物中多环芳烃的最大可允 许浓度 (maxim $\mu \mathrm{m}$ permissible concentrations, MPCs) 及可忽略浓度 (negligible concentrations, $\mathrm{NCs})^{[25-26]}$, 风 险商可定义为:

$$
R Q_{\mathrm{NCs}}=C_{\mathrm{PAHs}} / C_{\mathrm{QV}(\mathrm{NCs})}
$$


表 1 重金属潜在生态风险评价各指标及分级标准

Tab.1 Factors and corresponding classification criteria of potential ecological risk assessment of heavy metals

\begin{tabular}{|c|c|c|c|c|c|}
\hline 因素 & 范围 & 含义 & 因素 & 范围 & 含义 \\
\hline 污染因子 & $C_{f}^{i}<1$ & 低 & 污染度 & $C_{d}<8$ & 低 \\
\hline \multirow[t]{3}{*}{$C_{f}^{i}$} & $1 \leqslant C_{f}^{i}<3$ & 中等 & $C_{d}$ & $8 \leqslant C_{d}<16$ & 中等 \\
\hline & $3 \leqslant C_{f}^{i}<6$ & 高 & & $16 \leqslant C_{d}<32$ & 相当高 \\
\hline & $6 \leqslant C_{f}^{i}$ & 极高 & & $32 \leqslant C_{d}$ & 极高 \\
\hline 风险因子 & $E_{r}^{i}<40$ & 低 & & & \\
\hline \multirow[t]{4}{*}{$E_{r}^{i}$} & $40 \leqslant E_{r}^{i}<80$ & 中等 & 潜在风险指数 & $R I<150$ & 低 \\
\hline & $80 \leqslant E_{r}^{i}<160$ & 相当高 & $R I$ & $150 \leqslant R I<300$ & 中等 \\
\hline & $160 \leqslant E_{r}^{i}<320$ & 高 & & $300 \leqslant R I<600$ & 相当高 \\
\hline & $320 \leqslant E_{r}^{i}$ & 极高 & & $600 \leqslant R I$ & 极高 \\
\hline
\end{tabular}

式中, $C_{\mathrm{PAHs}}$ 表示多环芳烃的平均浓度, $C_{\mathrm{QV}(\mathrm{NCs})} 、 C_{\mathrm{QV}(\mathrm{MPCs})}$ 分别表示 $\mathrm{NCs} 、 \mathrm{MPCs}$ 浓度. 当污染物的浓度超过 MPCs 值时, 表明该污染物的生态风险较大, 对周围环境有较大影响; NCs 定义为 MPCs 的百分之一, 当污染 物浓度低于该值时,表明该污染物生态风险较低或不具有生态风险,污染物浓度在 NCs 和 MPCs 值中间时, 说明具有中等程度的生态风险. 由于没有关于多氯联苯的 MPCs 及 NCs 值, 其相应 NCs 及 MPCs 值可用采用 Long $^{[27]}$ 提出的生态风险效应低值和生态风险效应中值代替来进行评价, 当某一污染物浓度低于效应范围低 值( the effects range-low, ERL) 时, 表示较小的生态风险, 其生态风险效应低于 $10 \%$; 而当污染物浓度高于效 应范围中值 (the effects range-median, ERM) 时,表示该污染物具有很高的生态风险, 其风险效应大于 $50 \%$; 当污染物浓度在 ERL 和 ERM 之间时, 表示具有中等程度生态风险.

\section{2 实验结果与讨论}

\section{1 堆场淤泥基本性质}

堆场内淤泥颜色呈深褐色、弱酸性(表 2), 土壤容重为 $1.26 \sim 1.30 \mathrm{~g} / \mathrm{cm}^{3}$, 含水率为 $22 \% \sim 27 \%$, 烧失量 为 $3.76 \% \sim 4.70 \%$, 有机碳含量为 $0.67 \% \sim 1.12 \%$, 各堆场土壤粒径均以 $2 \sim 20 \mu \mathrm{m}$ 的粉粒为主.

表 2 疏浚堆场淤泥基本性质

Tab. 2 Basic characteristics of slurries in dredged sediment landfill sites

\begin{tabular}{|c|c|c|c|c|c|c|c|c|}
\hline \multirow{2}{*}{ 堆场 } & \multirow{2}{*}{$\mathrm{pH}$} & \multirow{2}{*}{ 容重/ $\left(\mathrm{g} / \mathrm{cm}^{3}\right)$} & \multirow{2}{*}{ 含水率/\% } & \multirow{2}{*}{ 有机碳/\% } & \multirow{2}{*}{ 烧失量/\% } & \multicolumn{3}{|c|}{ 土壤粒径 } \\
\hline & & & & & & $<2 \mu \mathrm{m} / \%$ & $2 \sim 20 \mu \mathrm{m} / \%$ & $>20 \mu \mathrm{m} / \%$ \\
\hline 白笔 & 6.30 & 1.30 & 22.30 & 0.88 & 4.50 & 11.62 & 73.37 & 15.01 \\
\hline 孔湾 & 6.33 & 1.26 & 26.82 & 0.67 & 3.76 & 23.84 & 75.89 & 0.27 \\
\hline 南庄 & 6.17 & - & - & 1.12 & 4.70 & 26.66 & 68.28 & 5.06 \\
\hline
\end{tabular}

\section{2 堆场内重金属含量分布及潜在生态风险评价}

巢湖南庄堆场内各重金属含量最高, 其次是太湖白施堆场, 太湖孔湾堆场内各重金属含量最低. 各重金 属含量在 3 个堆场内有相似的分布规律, 其中 $\mathrm{Zn} 、 \mathrm{Cr}$ 在各个堆场内含量最高, 两者总量分别占白施、孔湾和 南庄堆场金属含量的 60\%、58\% 和 69\% , 其余重金属含量由大到小表现为: $\mathrm{Ni}>\mathrm{Pb}>\mathrm{Cu}>\mathrm{As}>\mathrm{Cd}>\mathrm{Hg}$, 其中 $\mathrm{Cd}$ 和 $\mathrm{Hg}$ 总含量只占总重金属含量的约 $0.1 \%$. 方差分析结果表明, 除 $\mathrm{Cr}$ 和 $\mathrm{Ni}$ 在 3 个堆场淤泥内分布差异不明 显外,其余重金属含量在 3 个堆场内的分布均具有显著性差异. 依据土壤环境质量标准 GB $15618-$ $1995^{[28]}$, 孔湾堆场淤泥重金属含量均符合一级标准; 白笠堆场重金属 $\mathrm{As} 、 \mathrm{Cu} 、 \mathrm{Hg} 、 \mathrm{Zn}$ 含量均符合一级标准, $\mathrm{Cd} 、 \mathrm{Cr} 、 \mathrm{~Pb}$ 含量符合二级标准, $\mathrm{Ni}$ 则处于三级标准; 南庄堆场仅 $\mathrm{As}$ 符合一级标准, $\mathrm{Cu} 、 \mathrm{Hg} 、 \mathrm{~Pb}$ 符合二级标准, $\mathrm{Cd} 、 \mathrm{Cr} 、 \mathrm{Ni} 、 \mathrm{Zn}$ 均处于三级标准. 
表 3 疏浚堆场淤泥重金属含量及相关基准值“

Tab.3 Concentrations of heavy metals in dredged sediment landfill sites and guideline values of heavy metals

\begin{tabular}{|c|c|c|c|c|c|c|c|c|}
\hline \multirow{2}{*}{$\begin{array}{l}\text { 重金属/ } \\
(\mathrm{mg} / \mathrm{kg})\end{array}$} & \multirow{2}{*}{ 白暆堆场 } & \multirow{2}{*}{ 孔湾堆场 } & \multirow{2}{*}{ 南庄堆场 } & \multicolumn{3}{|c|}{ 土壤环境质量标准 } & \multicolumn{2}{|c|}{ 土壤背景值 } \\
\hline & & & & 一级 & 二级 & 三级 & 江苏省 & 安徽省 \\
\hline As & $11.67 \pm 0.34^{\mathrm{b}}$ & $9.86 \pm 0.12^{\mathrm{a}}$ & $13.64 \pm 0.04^{\mathrm{c}}$ & 15 & 40 & 40 & 10 & 9 \\
\hline $\mathrm{Cd}$ & $0.29 \pm 0.01^{\mathrm{b}}$ & $0.13 \pm 0.01^{\mathrm{a}}$ & $0.62 \pm 0.01^{\mathrm{c}}$ & 0.20 & 0.30 & 1.0 & 0.126 & 0.097 \\
\hline $\mathrm{Cr}$ & $107.07 \pm 16.35^{\mathrm{a}}$ & $74.77 \pm 1.69^{\mathrm{a}}$ & $227.77 \pm 98.26^{\mathrm{a}}$ & 90 & 150 & 300 & 77.8 & 66.5 \\
\hline $\mathrm{Cu}$ & $33.02 \pm 0.78^{b}$ & $23.64 \pm 0.62^{\mathrm{a}}$ & $35.34 \pm 0.77^{b}$ & 35 & 50 & 400 & 22.3 & 20.4 \\
\hline $\mathrm{Hg}$ & $0.097 \pm 0.022^{\mathrm{b}}$ & $0.045 \pm 0.004^{\mathrm{a}}$ & $0.25 \pm 0.00^{\mathrm{c}}$ & 0.15 & 0.30 & 1.5 & 0.289 & 0.033 \\
\hline $\mathrm{Ni}$ & $48.75 \pm 2.68^{\mathrm{a}}$ & $36.56 \pm 3.19^{\mathrm{a}}$ & $92.50 \pm 32.24^{\mathrm{a}}$ & 40 & 40 & 200 & 26.7 & 29.8 \\
\hline $\mathrm{Pb}$ & $39.18 \pm 1.06^{\mathrm{b}}$ & $31.31 \pm 0.73^{\mathrm{a}}$ & $64.64 \pm 0.86^{c}$ & 35 & 250 & 500 & 26.2 & 26.6 \\
\hline $\mathrm{Zn}$ & $87.08 \pm 2.63^{b}$ & $64.85 \pm 1.95^{\mathrm{a}}$ & $231.96 \pm 3.15^{\mathrm{c}}$ & 100 & 200 & 500 & 62.6 & 62 \\
\hline
\end{tabular}

* 不同字母表示不同堆场淤泥重金属含量在 $P<0.05$ 水平差异显著.

在巢湖南庄堆场内重金属污染因子较高, 其中 $\mathrm{Pb} 、 \mathrm{Cu} 、 \mathrm{As}$ 具有中等程度的污染因子, 而 $\mathrm{Zn} 、 \mathrm{Cr} 、 \mathrm{Ni}$ 以及 $\mathrm{Cd} 、 \mathrm{Hg}$ 污染因子均很高或者非常高; 白施及孔湾堆场的各个重金属污染因子则相对较低, 在白施堆场内, 除 $\mathrm{Hg}$ 的污染因子较低外,其余重金属均具有中等程度的污染因子,而在孔湾堆场内, Cr、As、 $\mathrm{Hg}$ 重金属污染因 子较低,其余重金属具有中等程度的污染因子,但污染程度均小于白施堆场 (图 1a). 对比重金属含量分布 发现, 虽然 $\mathrm{Cd} 、 \mathrm{Hg}$ 在淤泥内含量最低,但其污染程度更大, 尤其是在南庄堆场内, $\mathrm{Cd} 、 \mathrm{Hg}$ 具有非常高的污染 因子, 即使在白笔与孔湾堆场内, $\mathrm{Cd}$ 的污染因子也相对较高. 白暆、孔湾和南庄堆场重金属的污染度分别为 $11.42 、 7.80$ 和 30.03 , 重金属在孔湾堆场底泥内污染较低,在白笔堆场底泥内则有中等程度的污染, 而在南庄 堆场内则污染程度较高 (表 1 ).

巢湖南庄堆场淤泥内除 $\mathrm{Cd} 、 \mathrm{Hg}$ 金属风险因子很高外,其余重金属的风险因子均很低; 太湖白施堆场淤 泥内 $\mathrm{Cd}$ 具有中等程度的风险因子,其余重金属风险因子则很低,太湖孔湾堆场淤泥内所有金属污染因子均 很低, 但 $\mathrm{Cd} 、 \mathrm{Hg}$ 相对于其他金属则具有较高的风险因子值; $\mathrm{Cd} 、 \mathrm{Hg}$ 总风险因子在白施、孔湾和南庄堆场内分 别约占总风险因子的 $67 \% 、 55 \%$ 和 $89 \%$, 然而, 3 个堆场内含量较高的 $\mathrm{Zn} 、 \mathrm{Cr}$, 其总风险因子仅占重金属总风 险因子的约 3.6\%、4.3\% 和 1.9\% (图 1b). 这是因为重金属 $\mathrm{Hg} 、 \mathrm{Cd}$ 的毒性响应因子很大, 即使在较低含量的 情况下其风险也相对其余重金属要高, 而 Zn、Cr 则毒性相对较低, 因此即使含量很高其风险也较低. 白施、 孔湾和南庄重金属潜在生态危害指数分别为 $121.35 、 68.45$ 和 561.16 , 白施及孔湾两堆场内重金属的潜在生 态危害指数均很低, 而南庄堆场内重金属则对周围环境具有较高的潜在生态危害, 且危害主要来源于含量 较小的 $\mathrm{Cd}$ 和 $\mathrm{Hg}$.

\section{3 堆场内多环芳烃及多氯联苯含量分布及潜在生态风险评价}

多环芳烃 DahA、InP、Fla、Ant、Pyr 和 BghiP 在 3 个堆场内均有检出 (图 2a), 且检出含量最高的是 DahA 和 $\mathrm{InP}$, 两者总含量分别约占白施、孔湾和南庄堆场多环芳烃总含量的 76\%、63\% 和 $41 \%$, 对这 6 种多环芳烃 的方差分析结果显示, 除 Fla $\mathrm{Pyr}$ 分布具有显著性差异外,其余种类多环芳烃分布均不具有显著性差异. 巢 湖南庄堆场淤泥内的多环芳烃含量最高, 种类也最多, 共有 11 种, 其中 $\mathrm{BbF} 、 \mathrm{BaA} 、 \mathrm{Chr}$ 及 Flr 这 4 种多环芳烃 仅在南庄堆场淤泥内被检出, 但含量相对较低, 仅占总含量的 $13 \%$. 太湖孔湾堆场共检出多环芳烃 7 种, 太 湖白施堆场仅检出 6 种多环芳烃. 16 种多环芳烃总含量在 3 个堆场淤泥内由大到小表现为南庄堆场 $>$ 孔湾 堆场>白施堆场.

疏浚堆场淤泥内共监测到 9 种不同种类的多氯联苯 (图 2b) , 分别为 PCB8、PCB18、PCB28、PCB44、 PCB52、PCB77、PCB138、PCB195 和 PCB209. 其中 PCB77、PCB138、PCB195 和 PCB209 仅在巢湖南庄堆场淤 泥内被检出, 其余种类多氯联苯在 3 个堆场内均被检出,但所有种类多氯联苯在 3 个堆场内均不具有显著性 差异. 检出含量最高的是 PCB8, 在 3 个堆场淤泥内均约占多氯联苯总含量的 $90 \%$. 巢湖南庄堆场淤泥内多 氯联苯总含量最高, 达到 $3094 \mu \mathrm{g} / \mathrm{kg}$, 且检出多氯联苯种类也最多. 


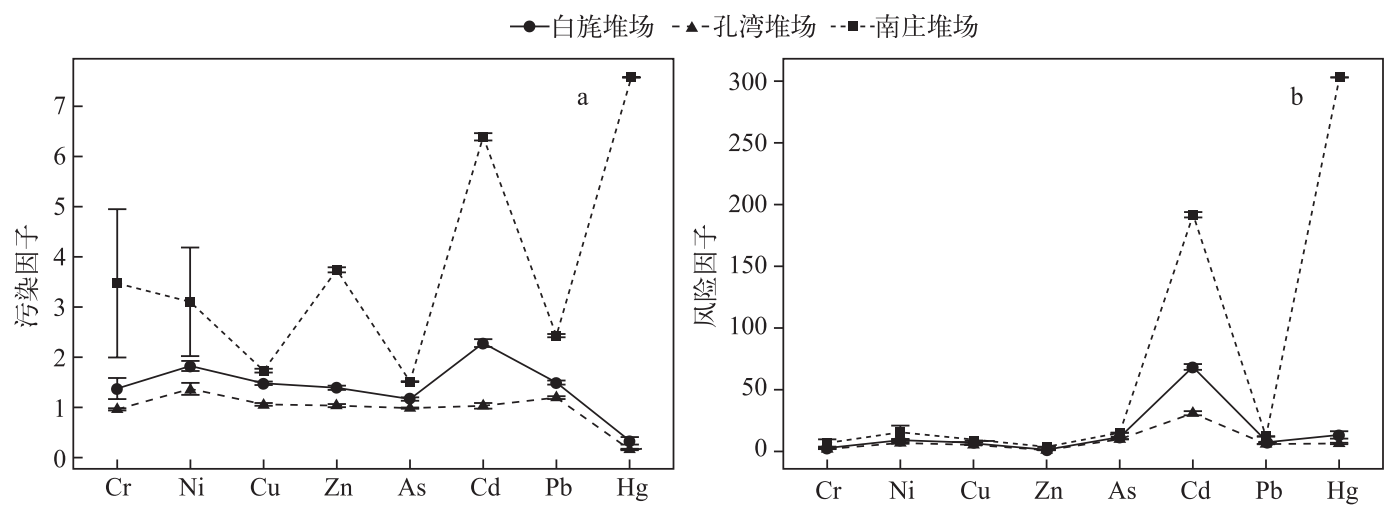

图 1 疏浚堆场淤泥内重金属潜在生态风险评价各指标值

Fig. 1 Potential ecological risk indexes of heavy metals in sludge in dredged sediments landfill sites
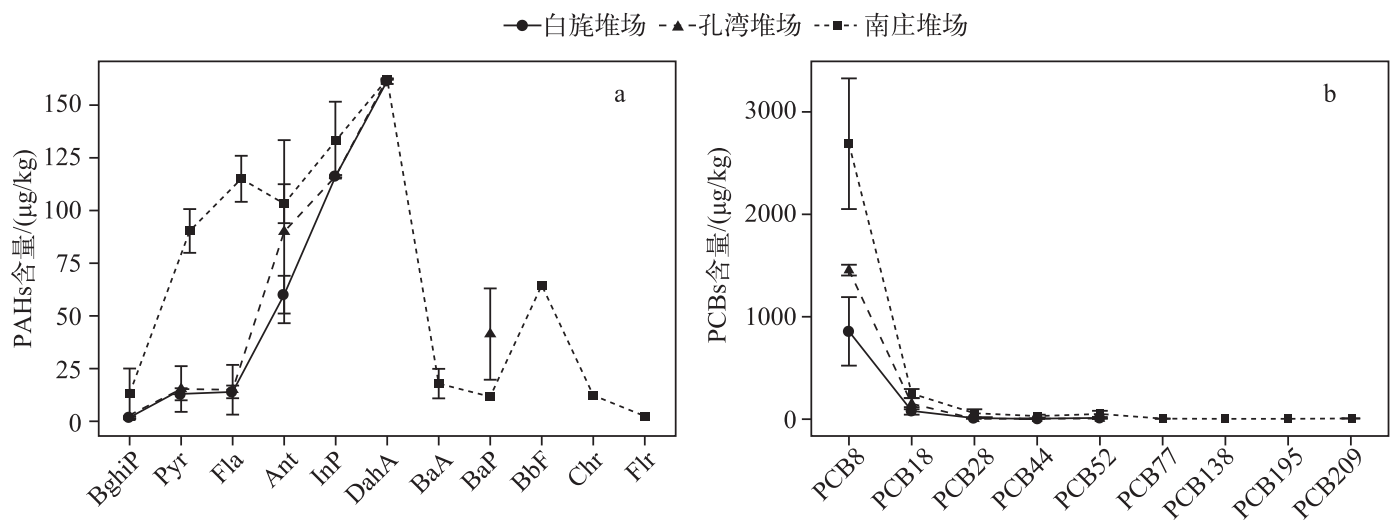

图 2 疏浚堆场淤泥内各多环芳烃 $(a)$ 及多氯联苯 $(b)$ 含量

Fig.2 Concentrations of PAHs(a) and $\operatorname{PCBs}(\mathrm{b})$ in sludge in dredged sediments landfill sites

表 4 是各多环芳烃及总多氯联苯的风险商值, 当 $R Q_{\mathrm{NCs}}<1$ 且 $R Q_{\mathrm{MPCs}}<1$ 时, 表示没有生态风险, 当 $R Q_{\mathrm{NCs}} \geqslant$ 1 且 $R Q_{\mathrm{MPCs}}<1$ 时, 表示具有中等程度的生态风险, 当 $R Q_{\mathrm{NCs}} \geqslant 1$ 且 $R Q_{\mathrm{MPCs}} \geqslant 1$ 时表示具有很高的生态风险. 对 比各堆场内多环芳烃及多氯联苯的生态风险商值可发现, 各堆场内多氯联苯的风险商值均很高, $R Q_{\mathrm{NCs}}$ 和 $R Q_{\mathrm{MPC}}$ 均大于 1 , 表明具有很高的生态风险; 而所有堆场淤泥内的多环芳烃的 $R Q_{\mathrm{MPC}}$ 值均小于 1 , 表明具有中 等程度风险或者不具有生态风险. 在巢湖南庄堆场内, 除 Flr、Chr、BaP 和 BghiP 不具有生态风险外, 其余 7 种检出的多环芳烃均具有中等程度的生态风险; 孔湾和白㔍堆场内, 除 Fla、Pyr 和 BghiP 不具有生态风险 外, 其余检出的多环芳烃均具有中等程度的生态风险. 在 3 个堆场中, Ant 和 DahA 的 $R Q_{\mathrm{Ncs}}$ 值均大于 50 , 表明 这 2 种多环芳烃相对于其他多环芳烃来说具有较大的环境风险.

\section{4 堆场淤泥与湖区底泥污染物负荷的比较}

本研究除 $\mathrm{Ni} 、 \mathrm{Hg}$ 表现出较明显的降低趋势外,其余重金属含量均与相应湖区内沉积物重金属含量分布 相当 ${ }^{[20,29]}$, 表明了重金属的保守性; 至于含量明显减少的 $\mathrm{Ni} 、 \mathrm{Hg}$, 可能是与 $\mathrm{Hg}$ 的挥发以及渗透流失有关. 相 对于梅梁湾湖区沉积物中的多环芳烃 ${ }^{[30]}, 16$ 种优先控制多环芳烃含量在疏浚淤泥中则表现出了明显的降 低趋势, 其可能原因是在堆场使用一段时间后, 随着淤泥脱水及不断氧化过程的进行, 多环芳烃会通过氧化 降解、挥发或生物累积等途径 ${ }^{[13,31]}$ 进行迁移转化, 因此疏浚底泥经堆放后, 淤泥内多环芳烃含量会降低. 值 得关注的是, 疏浚淤泥中的多氯联苯含量要远远大于湖泊底泥中多氯联苯含量 ${ }^{[30]}$, 显示了多氯联苯的持久 性以及易富集性. 
表 4 疏浚堆场淤泥内多环芳烃及多氯联苯风险商值”

Tab.4 Risk Quotients of PAHs and total PCBs in sludge in dredged sediments landfill sites

\begin{tabular}{|c|c|c|c|c|c|c|c|c|}
\hline \multirow{2}{*}{ 污染物 } & \multirow{2}{*}{$\begin{array}{c}\text { MPCs/ } \\
(\mu \mathrm{g} / \mathrm{kg})\end{array}$} & \multirow{2}{*}{$\begin{array}{c}\text { NCs/ } \\
(\mu \mathrm{g} / \mathrm{kg})\end{array}$} & \multicolumn{2}{|c|}{ 白暆堆场 } & \multicolumn{2}{|c|}{ 孔湾堆场 } & \multicolumn{2}{|c|}{ 南庄堆场 } \\
\hline & & & $R Q_{\mathrm{NCs}}$ & $R Q_{\mathrm{MPCs}}$ & $R Q_{\mathrm{NCs}}$ & $R Q_{\mathrm{MPCs}}$ & $R Q_{\mathrm{NCs}}$ & $R Q_{\mathrm{MPC}}$ \\
\hline Flr & 2600 & 26 & - & - & - & - & 0.09 & 0.00 \\
\hline Ant & 120 & 1.2 & 50.07 & 0.50 & 74.97 & 0.75 & 86.03 & 0.86 \\
\hline Fla & 2600 & 26 & 0.54 & 0.01 & 0.58 & 0.01 & 4.42 & 0.04 \\
\hline Pyr & 2600 & 26 & 0.49 & 0.00 & 0.59 & 0.01 & 3.47 & 0.03 \\
\hline $\mathrm{BaA}$ & 250 & 2.5 & - & - & - & - & 7.15 & 0.07 \\
\hline Chr & 10700 & 107 & - & - & - & - & 0.12 & 0.00 \\
\hline $\mathrm{BbF}$ & 2400 & 24 & - & - & - & - & 2.69 & 0.03 \\
\hline $\mathrm{BaP}$ & 2700 & 27 & - & - & 1.53 & 0.02 & 0.43 & 0.00 \\
\hline $\mathrm{InP}$ & 5900 & 59 & 1.97 & 0.02 & 1.97 & 0.02 & 2.26 & 0.02 \\
\hline DahA & 260 & 2.6 & 62.12 & 0.62 & 62.03 & 0.62 & 62.44 & 0.62 \\
\hline BghiP & 7500 & 75 & 0.02 & 0.00 & 0.02 & 0.00 & 0.18 & 0.00 \\
\hline 总 PCBs & $180^{\text {ERM }}$ & $22.7^{\text {ERL }}$ & 42.52 & 5.36 & 71.30 & 8.99 & 136.28 & 17.19 \\
\hline
\end{tabular}

*-表示该物质未检出.

重金属和多氯联苯等有毒物质不易被降解,而易于在堆场淤泥内累积, 从而对环境造成潜在的威胁, 尤 其是多氯联苯, 其含量均远远大于 ERM 值, 对环境具有很大的潜在生态风险, 应引起管理者的重视. 对于堆 场内的重金属污染物, 虽然 $\mathrm{Zn} 、 \mathrm{Cr}$ 含量高, 但其潜在生态风险却很低, 反而含量较低的 $\mathrm{Cd} 、 \mathrm{Hg}$ 却有较大的环 境风险. 因此, 对于堆场内污染物的潜在生态风险, 不仅应考虑其含量大小, 同时还应考虑到污染物的环境 化学行为和暴露迁移途径等因素. 堆场淤泥内污染物可通过各种方式向周围环境中扩散迁移从而对周围生 态系统产生影响: 污染物可溶解于降雨所产生的表面径流从而向周围水体中扩散, 或者直接渗透进人地下 水中 ${ }^{[11,32]}$; 对于挥发性的污染物质, 则可通过挥发作用进人空气中 ${ }^{[33]}$; 同时某些污染物也可通过生物累积 放大作用而进人生物体内 ${ }^{[13,34]}$. 湖泊底泥经疏浚后进人堆场, 将暴露在好氧的环境中, $\mathrm{pH}$ 也会随之降低, 这 种环境下淤泥中的一些重金属和有机物则更容易释放 ${ }^{[35]}$, 从而随降雨产生的表面径流进人周围水体或者直 接渗透进人地下水中, 对水体产生危害 ${ }^{[36]}$. 持久性有机污染物难以被降解, 且可通过生物累积作用进人生 物体内, 从而对生物体造成潜在的危害. 例如五里湖疏浚堆场植物水花生体内优先检测多环芳烃含量在堆 场使用 8 个月后增加了约 6 倍, 同时也检测到了一些在堆场使用前未检测到的多环芳烃 ${ }^{[13]}$. 因此, 对于疏浚 堆场内淤泥污染物, 不仅需要研究其内所含污染物的潜在生态风险, 同时还应对其迁移转化途径进行研究. 同时,疏浚底泥应及时进行处理,否则可能会对周围环境产生危害.

\section{3 结论}

疏浚堆场内淤泥中污染物对周围环境具有潜在的生态风险, 其中巢湖南庄堆场内淤泥内污染物含量最 高, 污染物种类也最多, 具有很大的环境风险; 而太湖白施及孔湾堆场淤泥污染物含量相对较低, 潜在生态 风险也相对较小. 对于堆场淤泥内重金属污染物, $\mathrm{Cd} 、 \mathrm{Hg}$ 含量仅约占淤泥内重金属总含量的 $0.1 \%$, 但两者 的潜在生态风险却最大, 对环境具有较大的潜在危害. 16 种优先检测多环芳烃中, DahA、Ant 具有较大的潜 在危害; 多氯联苯在淤泥内的含量非常高, 远大于 ERM 值, 对周围环境具有很高的生态风险, 应引起管理者 的重视. 另外, 相对于湖泊底泥, 疏浚淤泥经堆放后多环芳烃含量减小, 而重金属未表现出明显的降解趋势, 多氯联苯则在淤泥内有很高的积累. 对于堆场淤泥的堆放环境风险分析, 不仅应考虑污染物的含量, 同时还 考虑到污染物的扩散迁移途径及其环境化学行为, 在堆场淤泥进行后续利用时也要综合考虑有毒有害污染 物的生态风险; 另外堆场淤泥应尽快处置,尽量减少和避免对周围环境的影响.

\section{4 参考文献}

[ 1 ] BelHadjAli I, Lafhaj Z, Bouassida M et al. Characterization of Tunisian marine sediments in Rades and Gabes harbors. In- 
ternational Journal of Sediment Research, 2014, 29(3) : 391-401.

[ 2 ] Marmin S, Dauvin JC, Lesueur P. Collaborative approach for the management of harbour-dredged sediment in the Bay of Seine( France).Ocean \& Coastal Management, 2014, 102: 328-339.

[ 3 ] Ryding SO. Lake Trehörningen restoration project-Changes in water quality after sediment dredging. Hydrobiologia, 1982, 91/92(1) : 549-558.

[ 4 ] van der Does J, Verstraelen P, Boers P et al. Lake restoration with and without dredging of phosphorus-enriched upper sediment layers. Hydrobiologia, 1992, 233(1/2/3) : 197-210.

[ 5 ] Zhong Jicheng, Liu Guofeng, Fan Chengxin et al. Environmental effect of sediment dredging in lake( I ): the role of sediment dredging in reducing internal phosphorus release. J Lake Sci, 2009, 21(1): 84-93(in Chinese with English abstract). DOI: 10.18307/2009.0111. [ 钟继承, 刘国锋, 范成新等. 湖泊底泥疏浚环境效应: I. 内源磷释放控制作用. 湖泊科学, 2009, 21(1): 84-93.]

[ 6 ] Zhong Jicheng, Liu Guofeng, Fan Chengxin et al. Environmental effect of sediment dredging in lake( II ) : the role of sediment dredging in reducing internal nitrogen release. J Lake Sci, 2009, 21 (3): 335-344 (in Chinese with English abstract). DOI : 10.18307/2009.0304. [ 钟继承, 刘国锋, 范成新等. 湖泊底泥疏浚环境效应: II. 内源氮释放控制作用. 湖泊科学, 2009, 21(3): 335-344.]

[ 7 ] Zhang Xudong, Qi Jiying. Utilize of dredged sediment as resource. North Environment, 2005, 30(2) 48-50(in Chinese with English abstract). [张旭东, 祁继英.疏浚底泥的资源化利用. 北方环境, 2005, 30(2) : 48-50.]

[ 8 ] Zhu Guangwei, Chen Yingxu, Zhou Gendi et al. Characteristic of nutrient and polluting chemistry in dredged sludge. Plant Nutrition and Fertilizer Science, 2007, 7(3):311-317( in Chinese with English abstract). [ 朱广伟, 陈英旭, 周根娣等. 疏浚底泥的养分特征及污染化学性质研究. 植物营养与肥料学报, 2001, 7(3): 311-317.]

[ 9 ] Zhu Guangwei, Chen Yingxu, Wang Fengping et al. Application of sediment dredged in Grand Canal on agriculture. Agroenvironmental Protection, 2001, 20(2): 101-103 (in Chinese with English abstract). [ 朱广伟, 陈英旭, 王风平等. 城 市河道疏浚底泥农田应用的初步研究. 农业环境保护, 2001, 20(2): 101-103.]

[10] US and EPA. 16 priority pollutant polycyclic aromatic hydrocarbons(PAHs) 2008, Washington DC: US EPA.

[11] Stephens SR, Alloway BJ, Parker A et al. Changes in the leachability of metals from dredged canal sediments during drying and oxidation. Environmental Pollution, 2001, 114(3) : 407-413.

[12] Rumney HS, Bolam SG, Law RJ. Polycyclic aromatic hydrocarbons in sediments at dredged material disposal sites around England: Concentrations in 2013 and time trend information at selected sites 2008 - 2013. Marine Pollution Bulletin, $2015, \mathbf{9 2}(1 / 2): 180-185$.

[13] Zhang Lu, Fan Chengxin, Xian Qiming et al.Persistent organic pollutants(POPs) distribution and potential ecological risk on sediment in Lake Taihu and slurry stockyard. J Lake Sci, 2007, 19(1): 18-24 (in Chinese with English abstract). DOI : 10.18307/2007.0103. [张路, 范成新, 鲜启明等. 太湖底泥和疏竣堆场中持久性有机污染物的分布及潜在生 态风险. 湖泊科学, 2007,19 (1): 18-24.]

[14] Li Tao, Zhang Zhihong, Tang Baorong. Experimental study of retardant effect of clay barries on contaminants in a confineddisposal facility for dredged sediments from Taihu Lake. Rock and Soil Mechanics, 2012, 33(4) : 993-998(in Chinese with English abstract). [李涛, 张志红, 唐保荣. 太湖疏浚底泥堆场黏土防渗层阻隔污染物的试验研究. 岩土力学, $2012,33(4)$ : 993-998.]

[15] Yin Fucai, Zhang Zhiyuan. Survey of Chaohu Lake eutrophication research. J Lake Sci, 2003, 15 (4): 377-384 (in Chinese with English abstract). DOI: 10.18307/2003.0413. [殷福才, 张之源. 巢湖富营养化研究进展. 湖泊科学, $2003, \mathbf{1 5}(4)$ : 377-384.]

[16] Zhu Guangwei. Eutrophic status and causing factors for a large, shallow and subtropical Lake Taihu, China.J Lake Sci, 2008, 20 (1) : 21-26 (in Chinese with English abstract). DOI : 10.18307/2008.0103. [ 朱广伟. 太湖富营养化现状及原 因分析. 湖泊科学, 2008, 20(1): 21-26.]

[17] Li Congcong, Cheng Xiaoying, Zhang Guangsheng et al. A study on spatial and temporal variations of heavy metal contents in water body of Meiliang Bay in Taihu Lake. Shanghai Environmental Sciences, 2010, 29(5) : 185-191, 212(in Chinese with English abstract). [ 李聪聪, 成小英, 张光生等.太湖梅梁湾水体重金属浓度的时空变化研究. 上海环境科学, 2010, 29(5): 185-191, 212.]

[18] Du Chenchang, Liu Enfeng, Yang Xiangdong et al. Characteristics of enrichment and evaluation of anthropogenic pollution of heavy metals in the sediments of Lake Chaohu. J Lake Sci, 2012, 24(1) : 59-66( in Chinese with English abstract). 
DOI: $10.18307 / 2012.0108$. [杜臣昌, 刘恩峰, 羊向东等.巢湖沉积物重金属富集特征与人为污染评价. 湖泊科学, $2012, \mathbf{2 4}(1)$ : 59-66.

[19] Lu Rukun ed. Soil agricultural chemical analysis method. Beijing: Agricultural Scientech Press, 2000( in Chinese). [ 鲁 如坤. 土壤农业化学分析方法. 北京: 农业科技出版社, 2000.]

[20] Liu Cheng, Shao Shiguang, Fan Chengxin et al. Pollution status and risk assessment of heavy metal in the sediment of the severe polluted confluence area of Lake Chaohu. China Environment Science, 2014, 34(4) : 1031-1037(in Chinese with English abstract). [刘成, 邵世光, 范成新等.巢湖重污染汇流湾区沉积物重金属污染特征及风险评价. 中国环境 科学, 2014, 34(4): 1031-1037.]

[21] Teng Ying, Luo Yongming, Li Zhengao et al. Enchanced measures of indigenous microbial remediation in polychlorinated biphenyls polluted soils. Soils, 2006, 38(5): 645-651 (in Chinese with English abstract). [滕应, 骆永明, 李振高等. 多氯联苯复合污染土壤的土著微生物修复强化措施研究. 土壤, 2006, 38(5): 645-651.]

[22] Hakanson Lars. An ecological risk index for aquatic pollution control. A sedimentological approach. Water Research, 1980, 14(8): 975-1001.

[23] Zhao Qinna, Xu Qixin, Yang Kai. Application of potential ecological risk index in soil pollution of typical polluting industries.Journal of East China Normal University (Natural Science), 2005, (1):111-116(in Chinese with English abstract). [赵沁娜, 徐启新, 杨凯.潜在生态危害指数法在典型污染行业土壤污染评价中的应用. 华东师范大学学报(自然 科学版), 2005, (1) : 111-116.]

[24] China National Environmental Monitoring Centre. Background values of soil elements in China. Beijing: China Environmental Science Press, 1990(in Chinese). [中国环境监测总站. 中国土壤元素背景值. 北京: 中国环境科学出版 社, 1990.]

[25] Liu Minxia, Zhu Yindi, Zhang Miaomiao et al. Concentration, distribution, source, and risk assessment of PAHs and heavy metals in surface water from the Three Gorges Reservoir, China. Human and Ecological Risk Assessment, 2015,21 (6) : 1593-1607.

[26] Kalf DF, Crommentuijn T, van de Plassche EJ. Environmental quality objectives for 10 polycyclic aromatic hydrocarbons (PAHs). Ecotoxicology and Environmental Safety, 1997, 36(1) : 89-97.

[27] Long ER, Macdonald DD, Smith SL et al. Incidence of adverse biological effects within ranges of chemical concentrations in marine and estuarine sediments. Environmental Management, 1995, 19(1) : 81-97.

[28] Nanjing Institute of Environmental Sciences, MEP ed. Environmental quality standard for soil. National Bureau of Quality Inspection, 1995 (in Chinese). [ 国家环境保护局南京环境科学研究所.土壤环境质量标准. 国家质检总局, 1995.]

[29] Shi Zhifang. The characteristics of pollution and ecological risk assessment of heavy metals in sediment of Taihu Lake[ Dissertation]. Yangling: Northwest Agriculture and Forestry University, 2010(in Chinese with English abstract). [石志芳. 太湖沉积物中重金属污染特征及生态风险评价 [学位论文]. 杨凌:西北农林科技大学,2010.]

[30] Qiao Min, Huang Shengbiao, Zhu Yongguan et al. Ecological risk assessment of polycyclic aromatic hydrocarbons in sediments of Meiliang Bay, Taihu Lake. Asian Journal of Ecotoxicology 2007, 2(4): 456-463(in Chinese with English abstract). [乔敏, 黄圣彪, 朱永官等. 太湖梅梁湾沉积物中多环芳烃的生态和健康风险. 生态毒理学报, 2007,2 (4) : 456-463.]

[31] Eijsackers H, Bruggeman J, Harmsen J et al. Colonization of PAH-contaminated dredged sediment by earthworms. Applied Soil Ecology, 2009, 43(2/3): 216-225.

[32] Singh SP, Tack FMG, Gabriels D et al. Heavy metal transport from dredged sediment derived surface soils in a laboratory rainfall simulation experiment. Water, Air, and Soil Pollution, 2000, 118(1/2) : 73-86.

[33] Lü Jian, Huo Shouliang, Jing Yifeng et al. Research on pollutant diffusion control of contaminated dredged material on the mud dumping site. Journal of Beijing University of Technology, 2006, 32(3) : 229-234( in Chinese with English abstract). [吕鑑, 霍守亮, 荆一风等.环保疏浚底泥堆场污染物扩散防治研究. 北京工业大学学报, 2006, 32(3): 229-234.]

[34] Tang NH, Myers TE. PCB removal from contaminated dredged material. Chemosphere, 2002, 46(3) : 477-484.

[35] Almeida MSS, Borma LS, Barbosa MC. Barbosa, land disposal of river and lagoon dredged sediments. Engineering Geolo$g y, 2001, \mathbf{6 0}(1): 21-30$.

[36] Lions J, Guérin V, Bataillard P et al. Metal availability in a highly contaminated, dredged-sediment disposal site: Field measurements and geochemical modeling. Environmental Pollution, 2010, 158(9): 2857-2864. 